\title{
Polarization of Gravitational Waves from Binary Systems
}

\author{
L. Nyadzani* \\ Centre for Astro-Particle Physics (CAPP) and Department of Physics, University of \\ Johannesburg, Auckland Park 2006, South Africa \\ E-mail: lutendonyadzani@gmail.com

\section{S. Razzaque} \\ Centre for Astro-Particle Physics (CAPP) and Department of Physics, University of \\ Johannesburg, Auckland Park 2006, South Africa \\ E-mail: srazzaque@uj.ac.za
}

Gravitational waves (GWs) are one of the key predictions of Einstein's theory of general relativity (GR). Scientists have been looking for evidence of GWs since their prediction by Einstein in 1916. The first direct detection was achieved by LIGO in 2015, 100 years after their prediction, from merging of two black holes in a binary into one. According to GR gravitational waves have two independent polarization states. In this paper, we study the power radiated and the strain of GW along the two polarization states from a compact binary system with its general orbital properties.

High Energy Astrophysics in Southern Africa - HEASA2018

1-3 August, 2018

Parys, Free State, South Africa

${ }^{*}$ Speaker. 


\section{Introduction}

Gravitational waves are one of the key predictions of Einstein's theory of general relativity (hereafter GR) [1,2]. In GR, accelerated massive bodies generate ripples in spacetime. These ripples radiate away from their source at the speed of light [3]. The same way the electromagnetic radiation carry information about their origin, GWs also carry information about their origin. The first indirect evidence of the existence of GW was a binary system discovered by Hulse and Taylor, known as PSR 1913+16 [4]. They observed that the orbital period of this system was decaying. This decay was consistent with the expected orbital decay of a system emitting gravitational radiation as predicted by the standard quadrupole formula in GR [5].

It was not until 2015 when the first direct detection of GWs (GW150914) was achieved by LIGO. This event was due to inspiraling orbit and merger of two black holes [6]. Since the GW150914 event, there have been 5 more confirmed detections made by LIGO and VIRGO namely GW151226, GW170104, GW170608, GW170814 and GW170817 [6-11]. With these detections, scientists have been able to probe some fundamental physics in the highly dynamical and strongfield regime of gravity, as predicted by the GR using GW signals. One of the key tools used to study GWs is the polarization states of these waves. In general, alternative theories of gravity predict up to six polarization states, namely plus, cross, breathing, longitudinal, vector- $x$ and vector- $y$, assuming that the GW is propagating in the $z$ direction [12]. However in GR, only two states of polarizations are allowed, the plus and the cross modes. In this paper, only these two independent polarizations from GR will be discussed.

This paper starts with an introduction to the theory of GW generation and propagation in spacetime. Then review the polarization states of GW as described by GR and finally study the GW power generated and strain of this wave in spacetime for two compact objects spiraling in an orbit with general configuration.

\section{GW Theory}

In the theory of general relativity, the Einstein's field equations are given by,

$$
R_{\mu v}-\frac{1}{2} g_{\mu v} R=-8 \pi G T_{\mu v}
$$

We solve equation (2.1) by assuming the spacetime far away from the source to be a Minkowski spacetime $\eta_{\mu v}$ (flat). Let the GW passing through the observer be represented by the perturbation $h_{\mu \nu}$ on a background spacetime $\eta_{\mu \nu}$. The spacetime metric $g_{\mu v}$ in (2.1) becomes $g_{\mu v}=\eta_{\mu \nu}+$ $h_{\mu \nu}$. Expanding (2.1) in powers of $h_{\mu v}$ and their derivatives $\left(\partial_{\mu} h_{\mu v}\right)$ under the harmonic gauge condition, we obtain the following wave equation [3].

$$
\square \bar{h}_{\mu v}=-16 \pi G T_{\mu v}
$$

where $\bar{h}_{\mu v}=h_{\mu v}-\frac{1}{2} \eta_{\mu v} h$, is "the trace-reversed tensor" of $h_{\mu v}$ 
In vacuum, where there is no matter present we have $T_{\mu \nu}=0$, and we have

$$
\square \bar{h}_{\mu \nu}=0
$$

The above equation has a plane wave solution,

$$
\bar{h}_{\mu v}=h_{\mu v}=a e_{\mu v} \cos (\omega t-\mathbf{k} \cdot \mathbf{x})
$$

Here $e_{\mu \nu}$ is the polarization unit tensor, $a$ is the GW amplitude, $\omega$ is the orbital angular frequency, $\mathbf{k}$ is the wave vector, and $\mathbf{x}$ is the position. Solving equation (2.3) with the above plane wave we find that there are only two independent components of $e_{\mu v}$, namely plus $\left(e^{+}\right)$and cross $\left(e^{\times}\right)$.

Equation (2.2) can be evaluated to find the power radiated by a system of masses undergoing arbitrary motion. Using quadrupole approximation, the power radiated into the solid angle $d \Omega$ with polarization $e_{i j}$ is [13],

$$
\frac{d E}{d t d \Omega}=\frac{G}{8 \pi c^{5}}\left(\frac{d^{3} Q_{i j}}{d t^{3}} e_{i j}\right)^{2}
$$

where $Q_{i j}$ is the quadrupole moment tensor given by

$$
Q_{i j}=\sum_{\alpha} m_{\alpha i} m_{\alpha j}
$$

and $\alpha=1,2$

\section{GW Polarization}

Consider a GW with orthonormal coordinates constructed by three unit vectors $\hat{k}, \hat{w}_{\theta}$ and $\hat{w}_{\phi}$. Let $\hat{k}$ be the direction of propagation of the GW such that unit vectors $\hat{w}_{\theta}$ and $\hat{w}_{\phi}$ are orthogonal to the propagation of this GW. In this coordinate system, the two polarization modes are defined as,

$$
\begin{aligned}
& e^{+}=\hat{w}_{\theta} \otimes \hat{w}_{\theta}-\hat{w}_{\phi} \otimes \hat{w}_{\phi} \\
& e^{\times}=\hat{w}_{\theta} \otimes \hat{w}_{\phi}+\hat{w}_{\phi} \otimes \hat{w}_{\theta}
\end{aligned}
$$

and are represented graphically in Figure 1.

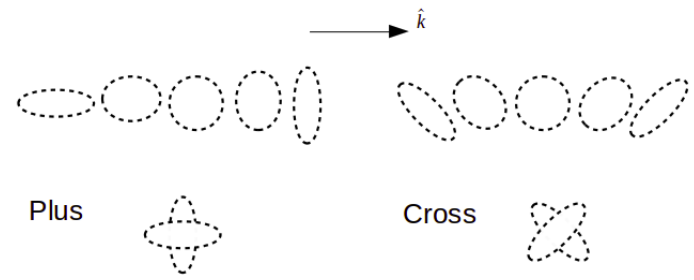

Figure 1: The effect of the plus and cross polarizations on a ring of particles in space. 
If we consider an observer at a distance $\mathbf{R}$ from the source with coordinates $\hat{x}=(1,0,0), \hat{y}=(0,1,0)$ and $\hat{z}=(0,0,1)$. Then the coordinate systems of the wave and observer are related by,

$$
\begin{aligned}
\hat{w}_{\theta} & =\hat{x} \cos \theta \cos \phi+\hat{y} \cos \theta \sin \phi-\hat{z} \sin \theta \\
\hat{w}_{\phi} & =-\hat{x} \sin \phi+\hat{y} \cos \phi \\
\hat{k} & =\hat{x} \sin \theta \cos \phi+\hat{y} \sin \theta \sin \phi+\hat{z} \cos \theta
\end{aligned}
$$

where $\theta$ and $\phi$ are the conventional polar coordinates, as shown in Figure 2.

\subsection{Power}

We now consider a binary system of masses $m_{1}$ and $m_{2}$ in a closed orbit around a common center of mass with eccentricity $e$ and semi-major axis $a$ and the observer is located as described above and in Figure 2. The total power radiated into the cone $d \Omega$ is given by equation (2.4).

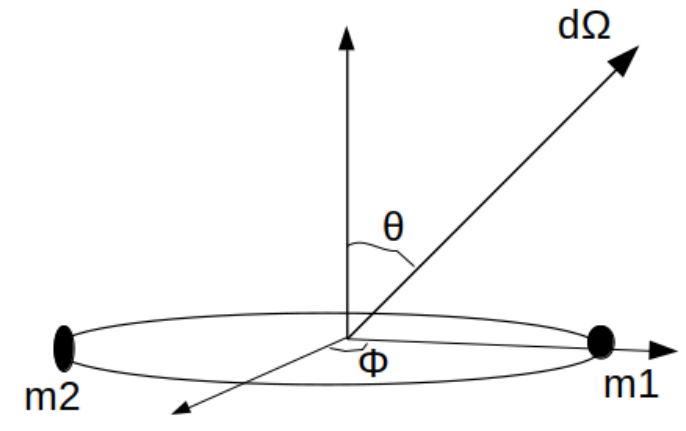

Figure 2: Binary system consisting of two masses $m_{1}$ and $m_{2}$ orbiting around a common center of gravity with semimajor axis $a$ and the orbital angular frequency $\omega$.

Powers radiated into the two polarization components are given by [14],

$$
\begin{aligned}
\frac{d P_{+}}{d \Omega} & =\frac{G^{4}}{\pi c^{5}} \frac{m_{1}^{2} m_{2}^{2}\left(m_{1}+m_{2}\right)}{a^{5}\left(1-e^{2}\right)^{7 / 2}}\left[\left(\frac{1}{2}+\frac{99}{64} e^{2}+\frac{51}{256} e^{4}\right)\left(1+\cos ^{4} \theta\right)+\left(1+\frac{95}{32} e^{2}+\frac{47}{128} e^{4}\right) \cos ^{2} \theta\right. \\
& \left.+\left(\frac{13}{32} e^{2}+\frac{1}{16} e^{4}\right)\left(1-\cos ^{4} \theta\right) \cos 2 \phi-\frac{25}{512} e^{4}\left(1+\cos ^{2} \theta\right)^{2} \cos 4 \phi\right] \\
\frac{d P_{\times}}{d \Omega} & =\frac{G^{4}}{\pi c^{5}} \frac{m_{1}^{2} m_{2}^{2}\left(m_{1}+m_{2}\right)}{a^{5}\left(1-e^{2}\right)^{7 / 2}}\left[\left(2+\frac{97}{16} e^{2}+\frac{49}{64} e^{4}\right) \cos ^{2} \theta+\frac{25}{128} e^{4} \cos ^{2} \theta \cos 4 \phi\right]
\end{aligned}
$$

Assuming that the emission of GW is symmetric over $\phi$ we can integrate equation (3.3) and (3.4) over $\phi(0,2 \pi)$ to get power radiated as a function of the inclination angle $\theta(0, \pi)$, eccentricity $e(0,1)$, masses $\left(m_{1}\right.$ and $\left.m_{2}\right)$, and semi-major axis $a$,

$$
\begin{aligned}
& P_{+}=\frac{G^{4}}{\pi c^{5}} \frac{m_{1}^{2} m_{2}^{2}\left(m_{1}+m_{2}\right)}{a^{5}\left(1-e^{2}\right)^{7 / 2}}\left[\left(\frac{1}{2}+\frac{99}{64} e^{2}+\frac{51}{256} e^{4}\right)\left(\cos \theta+\frac{1}{5} \cos ^{5} \theta\right)+\frac{1}{3}\left(1+\frac{95}{32} e^{2}+\frac{47}{128} e^{4}\right) \cos ^{3} \theta\right] \\
& P_{\times}=\frac{G^{4}}{\pi c^{5}} \frac{m_{1}^{2} m_{2}^{2}\left(m_{1}+m_{2}\right)}{3 a^{5}\left(1-e^{2}\right)^{7 / 2}}\left[\left(2+\frac{97}{16} e^{2}+\frac{49}{64} e^{4}\right) \cos ^{3} \theta\right]
\end{aligned}
$$


In Figure 3 we plot these powers as different functions of parameters.
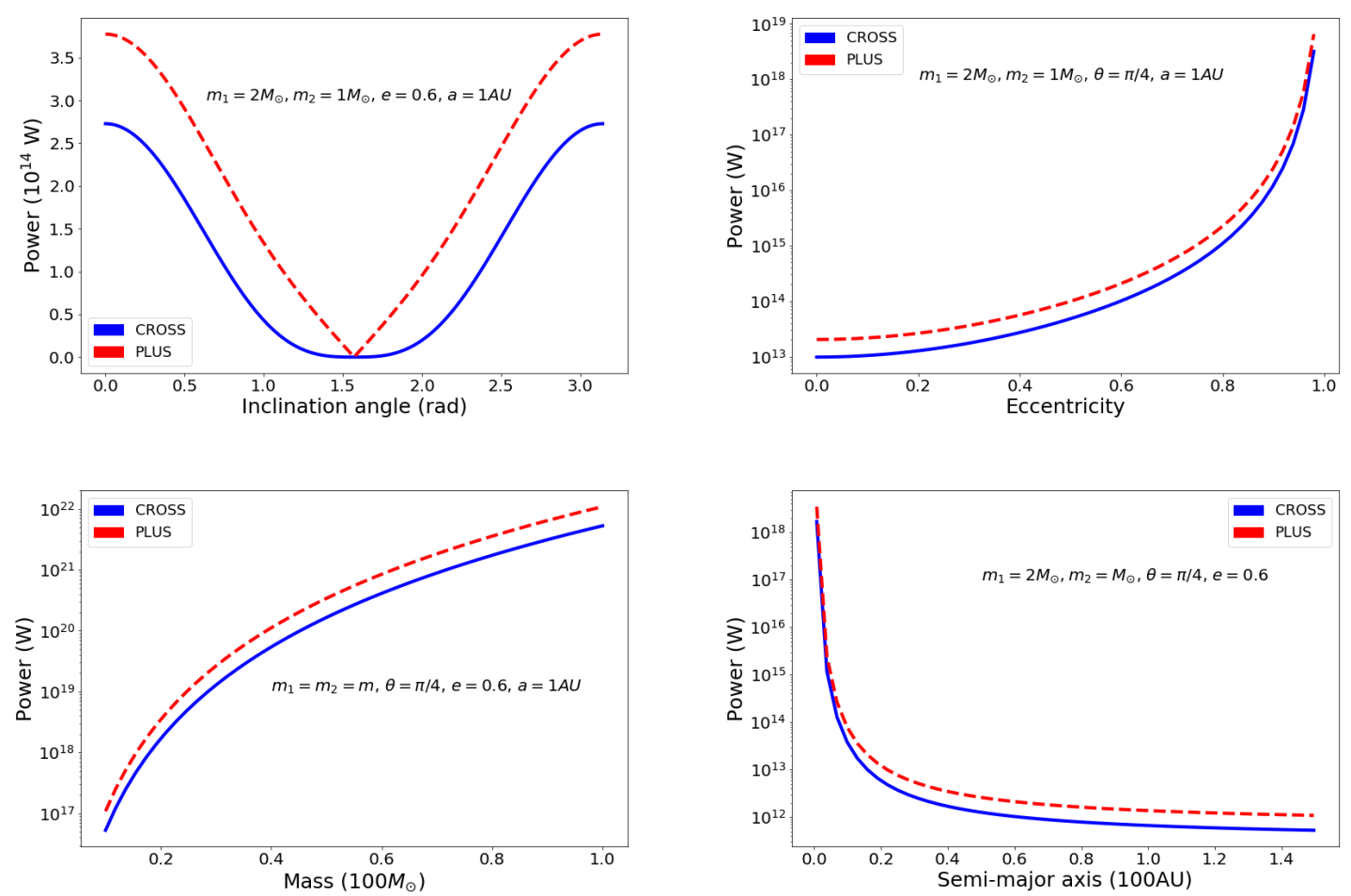

Figure 3: Power radiated into plus $(+)$ (red dashed line) and cross $(\times)$ (blue solid line) shows polarization components. Top left panel shows power as a function of the inclination angle $(0, \pi)$; top right panel shows power radiated as a function of the eccentricity $(e)$; bottom left panel shows power as a function of the mass and bottom right panel shows power as a function of the semi-major axis $(a)$.

\subsection{GW Strain}

If one solves the Einstein's field equation by assuming a small spacetime perturbation $\left(h_{\mu v}\right)$ on a flat spacetime background $\left(\eta_{\mu \nu}\right)$ at a distance $r$ from the source, one obtains that, the disturbance in space is given by the following formula [3],

$$
h_{i j}=\frac{1}{r} \ddot{Q}_{i j}
$$

where $r$ is the distance form the source to the observer and $Q_{i j}$ is the quadrupole moment. The polarization tensor $e_{i j}$ has only two independent components, the plus and cross polarization, and the strains for these components are defined as

$$
\begin{aligned}
& h_{+}=h_{i j} \times e^{+} \\
& h_{\times}=h_{i j} \times e^{\times}
\end{aligned}
$$


Calculating $Q_{i j}$ for a system described in Figure 2 using equation (2.5) and differentiate twice with respect to time, as required by equation (3.7), we obtain the following,

$$
\ddot{Q}_{i j}=\left[\begin{array}{ccc}
e \cos ^{3} \psi-2 \sin ^{2} \psi+1 & \left(e \cos ^{2} \psi+e+2 \cos \psi\right) \sin \psi & 0 \\
\left(e \cos ^{2} \psi+e+2 \cos \psi\right) \sin \psi & -\left(e^{2}+e \cos ^{3} \psi+\cos \psi-2 \sin ^{2} \psi+1\right) & 0 \\
0 & 0 & 0
\end{array}\right]
$$

where $\psi$ is the retarded position of the system. Having obtained $\ddot{Q}_{i j}$ we substitute (3.7) into (3.8) to obtain the following,

$$
\begin{aligned}
& h_{+}= \frac{2 G^{2} m_{1} m_{2}}{a c^{4} r\left(e^{2}-1\right)}\left(-\left(\sin ^{2} \phi \cos ^{2} \theta-\cos ^{2} \phi\right)\left(e^{2}+e \cos ^{3} \psi+e \cos \psi-2 \sin ^{2} \psi+1\right)\right. \\
&-\left(\sin ^{2} \phi-\cos ^{2} \phi \cos ^{2} \theta\right)\left(e \cos ^{3} \psi-2 \sin ^{2} \psi+1\right) \\
&\left.+2\left(\cos ^{2} \theta+1\right)\left(e \cos ^{2} \psi+e+2 \cos \psi\right) \sin \phi \sin \psi \cos \phi\right) \\
& h_{\times}=-\frac{2 G^{2} m_{1} m_{2}}{a c^{4} r\left(e^{2}-1\right)}\left(e^{2} \sin (2 \phi)+\frac{e}{2} \sin (2 \phi-3 \psi)+\frac{5 e}{2} \sin (2 \phi-\psi)+2 \sin (2 \phi-2 \psi)\right) \cos \theta
\end{aligned}
$$

Equations (3.9) and (3.10) show the relationship between GW strain and the four parameters namely, mass, distance traveled, eccentricity, inclination angle, semimajor axis and the retarded position. These are general equations for arbitrary orbital parameters.

From here on we will study circular systems by letting $e=0$ in equation (3.9) and (3.10) which give,

$$
\begin{aligned}
& h_{+}=-\frac{2 G^{2} m_{1} m_{2}}{r a c^{4}}\left(1+\cos ^{2} \theta\right) \cos (2 \psi-2 \phi) \\
& h_{\times}=-\frac{4 G^{2} m_{1} m_{2}}{r a c^{4}} \cos \theta \sin (2 \psi-2 \phi)
\end{aligned}
$$

It is better to write (3.11) and (3.12) in terms of orbital frequency $\omega$, and the semimajor axis is related to the orbital period by Kepler's law of orbits. Kepler's law of orbits

$$
T=\frac{2 \pi}{\omega}=2 \pi \sqrt{\frac{a^{3}}{G\left(m_{1}+m_{2}\right)}}
$$

Then

$$
a=\frac{G^{1 / 3}\left(m_{1}+m_{2}\right)^{1 / 3}}{\omega^{2 / 3}}
$$

Substituting (3.13) in (3.11) and (3.12) we obtain the following,

$$
\begin{aligned}
& h_{+}=-\frac{2 G^{5 / 3} m_{1} m_{2} \omega^{2 / 3}}{r c^{4}\left(m_{1}+m_{2}\right)^{1 / 3}}\left(1+\cos ^{2} \theta\right) \cos (2 \psi-2 \phi) \\
& h_{\times}=-\frac{4 G^{5 / 3} m_{1} m_{2} \omega^{2 / 3}}{r c^{4}\left(m_{1}+m_{2}\right)^{1 / 3}} \cos \theta \sin (2 \psi-2 \phi)
\end{aligned}
$$


Equations (3.14) and (3.15) are valid for objects moving with very small speed compared to the speed of light. The semimajor axis decreases slowly, and the orbital frequency can be taken to be constant over a long period of time. The orbital angular frequency is given by, $\omega=d \psi / d t$, then $\psi=\omega t$, assuming $\omega$ to be constant with time. We define new quantities $A_{+}, A_{\times}, g_{+}$and $g_{\times}$such that $h_{+}=A_{+} g_{+}$and $h_{\times}=A_{\times} g_{\times}$, where,

$$
\begin{aligned}
A_{+} & =\frac{2 G^{5 / 3} \mathscr{M}^{5 / 3} \omega^{2 / 3}}{r c^{4}}\left(1+\cos ^{2} \theta\right) \\
A_{\times} & =\frac{4 G^{5 / 3} \mathscr{M}^{5 / 3} \omega^{2 / 3}}{r c^{4}} \cos \theta \\
g_{+} & =\cos (2 \omega t-2 \phi) \\
g_{\times} & =\sin (2 \omega t-2 \phi)
\end{aligned}
$$

Here $\mathscr{M}=\left(m_{1} m_{2}\right)^{3 / 5} /\left(m_{1}+m_{2}\right)^{1 / 5}$ is the chirp mass, $\left(A_{+}, A_{\times}\right)$are the amplitude of the GW along the two polarizations, and $\left(g_{+}, g_{\times}\right)$are the GW sinusoidal waveform. Equations (3.18) and (3.19) show that the GW frequency is twice that of the orbital frequency and the GW amplitude falls off as $1 / r$ from equations (3.16) and (3.17). Thus, the effect of GW on spacetime decreases as the wave propagate away from the source. When the angle of inclination $\theta$ is zero or multiples of $\pi$ the plus and the cross amplitudes are equal. If the observer is a detector such as the LIGO and VIRGO, the orbital frequency can be determined from their data and the chirp mass can be calculated using relation [15],

$$
\mathscr{M}=\left(\frac{5}{96 \pi^{8 / 3} f^{11 / 3}} \partial_{t} f\right)^{3 / 5}
$$

Once the polarization modes are separated such that the inclination angle can be constrained, equations (3.16) and (3.17) can be used to constrain the distance to the source. For a system with $m_{1}=2 M_{\odot}, m_{2}=1.5 M_{\odot}, f=10^{-5} \mathrm{~Hz}$ and $100 \mathrm{kLy}$ from the earth (size of our galaxy), (3.16) and (3.17) give the amplitude to be $3.35 \times 10^{-24}$ for both $A_{+}$and $A_{\times}$with the inclination $(\theta=\pi)$. If the inclination angle is not a multiple of $\pi$ the two amplitudes are not equal. For example for a system described above with $\theta=0.4 \pi A_{+}$is twice $A_{\times}$and when the inclination $\theta$ is $\pi / 2$ the $A_{\times}$ vanishes and only $A_{+}$remains.

\section{Summary}

In this paper we have calculated how the power radiated by a binary system in the form of GW into the plus and cross polarizations is dependent on the masses, eccentricity, semi-major axis and the angle of inclination with respect to the observer. Studying GW polarization states is important in determining properties of the systems that generate them, such as, the inclination angle relative to the observer. Figure 2 shows that, power radiated into the plus polarization is more than that radiated into the cross polarization for $\theta \neq \pi / 2$. The GW strain hold information about the origin of the wave, such as the inclination angle, mass, orbital frequency and the distance traveled. If the amplitude of the wave is determined from the detectors such as LIGO, it can be used to determine some of the system properties using equations (3.16) and (3.17). 


\section{Acknowledgement}

This work was supported by grants from the National Research Foundation (NRF) South Africa.

\section{References}

[1] A. Einstein, Näherungsweise integration der feldgleichungen der gravitation. sitz.-ber. $k$, Preu $\beta$. Akad. Wiss 688 (1916) .

[2] A. Einstein, Über gravitationswellen, Sitzungsberichte der Königlich Preußischen Akademie der Wissenschaften (Berlin), Seite 154-167. (1918) .

[3] S. M. Carroll, Lecture notes on general relativity, arXiv preprint gr-qc/9712019 (1997) .

[4] R. A. Hulse and J. H. Taylor, Discovery of a pulsar in a binary system, The Astrophysical Journal 195 (1975) L51-L53.

[5] C. Sivaram, The Hulse-Taylor binary pulsar PSR 1913+ 16., Bulletin of the Astronomical Society of India 23 (1995) 77-83.

[6] B. P. Abbott, R. Abbott, T. Abbott, M. Abernathy, F. Acernese, K. Ackley et al., Observation of gravitational waves from a binary black hole merger, Physical Review Letters 116 (2016) 061102.

[7] B. P. Abbott, R. Abbott, T. D. Abbott, F. Acernese, K. Ackley, C. Adams et al., GW170608: Observation of a 19 Solar-mass Binary Black Hole Coalescence, ApJL 851 (Dec., 2017) L35, [1711.05578].

[8] B. P. Abbott, R. Abbott, T. Abbott, F. Acernese, K. Ackley, C. Adams et al., GW170817: observation of gravitational waves from a binary neutron star inspiral, Physical Review Letters 119 (2017) 161101.

[9] B. Abbott, R. Abbott, T. Abbott, F. Acernese, K. Ackley, C. Adams et al., GW170104: observation of a 50-solar-mass binary black hole coalescence at redshift 0.2, Physical Review Letters 118 (2017) 221101.

[10] B. P. Abbott, R. Abbott, T. Abbott, M. Abernathy, F. Acernese, K. Ackley et al., GW151226: Observation of gravitational waves from a 22-solar-mass binary black hole coalescence, Physical Review Letters 116 (2016) 241103.

[11] B. Abbott, R. Abbott, T. Abbott, M. Abernathy, F. Acernese, K. Ackley et al., Binary black hole mergers in the first Advanced LIGO observing run, Physical Review X 6 (2016) 041015.

[12] M. Isi, M. Pitkin and A. J. Weinstein, Probing dynamical gravity with the polarization of continuous gravitational waves, Physical Review D 96 (2017) 042001.

[13] P. Peters and J. Mathews, Gravitational radiation from point masses in a keplerian orbit, Physical Review 131 (1963) 435.

[14] P. C. Peters, Gravitational radiation and the motion of two point masses, Physical Review 136 (1964) B1224.

[15] N. Bishop, Current status of gravitational wave observations, PoS HEASA2017 (2018) 001. 